\title{
Simulation of the Long-QT Syndrome in a Model of Human Myocardium
}

\author{
G Seemann $^{1}$, DL Weiß ${ }^{1}$, FB Sachse ${ }^{2}$, O Dössel $^{1}$ \\ ${ }^{1}$ Institute for Biomedical Engineering, University Karlsruhe (TH), Germany \\ ${ }^{2}$ Nora Eccles Harrison Cardiovascular Research and Training Institute, University of Utah, USA
}

\begin{abstract}
Regional heterogeneity of electrophysiological properties within the human ventricular wall is based on changes in ion channel kinetics and densities. This leads to a dispersion of repolarization, which causes the positive $T$ wave in the ECG. Due to genetic defects, diseases, or drugs, which causes e.g. long-QT syndrome, the physiological heterogeneous properties get out of tune.

The aim of this work is to investigate the long$Q T$ syndrome in a realistic model of human ventricular anatomy and electrophysiology. An anisotropic, threedimensional, heterogeneous model is constructed, which incorporates an electrophysiological model describing complex ionic processes and a bidomain excitation model. LQT1, LQT2, and LQT3 where integrated in this model.

The results show that this type of model is capable not only to simulate the electrophysiology but also pathological cases. The features of the long-QT syndrome are mainly characterized correctly in the simualtions.
\end{abstract}

\section{Introduction}

Heterogeneity of ion channel expression is present in mammalian myocardium [1]. Three populations of cell types can be distinguished: endocardial, mid-myocardial (M), and epicardial cells. They differ with regard to their electrophysiology, response to pharmacologic agents, and in expression of pathology. M cells are located in the deep subendocardium in the human left ventricular free wall.

The electrical heterogeneity of these three cell types is characterized by variations of the density and kinetics of the transient outward potassium current $I_{t o}$, the slow-delayed rectifier potassium current $I_{K s}$, the potassium inward rectifier current $I_{K 1}$, the late component of the sodium current $I_{N a, L}$, and the sodium-calcium exchanger $I_{\mathrm{NaCa}}$. This leads transmural to different action potential durations (APD) and thereby to a dispersion of repolarization inside the ventricular wall, which is the cause for the positive $\mathrm{T}$ wave in the electrocardiogram (ECG). The complex interaction of the heterogeneous characteristics can be disturbed by genetic defects, diseases, or drugs.
One of the most investigated pathologies due to genetic defect is the long-QT syndrome (LQTS). It is caused by a change in the dispersion of ventricular repolarization and is characterized by changes in the morphology of the ECG during the QT interval and the T wave [1]. The QT interval is always extended and the $\mathrm{T}$ wave is broadened and enlarged in amplitude in some cases. The most common types of LQTS are LQT1, LQT2, and LQT3, where the characteristics of $I_{K s}$, the rapid-delayed rectifier current $I_{K r}$, and $I_{N a, L}$ are altered, resp..

The cause of the LQT1 syndrome is the loss of the $I_{K s}$ channel function due to a defect of channel gene KvLQT1 [2]. This loss increased the APD homogeneously in all cell types, since $I_{K r}$ is distributed homogeneously inside the ventricular wall as the remaining repolarization current. Besides the prolongation of the QT interval only small changes of the $T$ wave morphology is visible in the ECG.

For the LQT2 syndrome a defect in channel gene HERG is responsible for the $I_{K r}$ channel to lose its capability to let $K^{+}$ions pass [3]. Due to the heterogeneous distribution of the remaining repolarization current $I_{K s}$ the APD of the M cells is more extended than the APD of endo- or epicardial cells. This results apart from QT interval prolongation in larger amplitudes and wider characteristics of the $\mathrm{T}$ wave morphology.

A disturbance of the inactivating process of the $I_{N a}$ channel due to a defect in channel gene SCN5A is the reason for LQT3 [3]. During repolarization phase 2 the mutant $I_{N a}$ channel re-opens partly, which does not happen in physiological cases. The APD of all cell types is prolonged but to largest extend in $\mathrm{M}$ cells because of the already longer plateau phase. This leads to the prolongation of the QT interval and a widening and enlargement of the amplitude of the $\mathrm{T}$ wave.

Measured transmural ECGs of LQT1 as well as LQT2 and LQT3 are presented in [2] and [3] resp., where the three LQTS are modeled pharmacologically.

The aim of this work is to investigate the mechanisms and effects of these three types of LQTS in a realistic model of the human myocardium by assessing single cell simulations and transmural ECGs of coupled cells using detailed modeling of the electrophysiology. 


\section{Methods}

The heterogeneous and anisotropic three-dimensional computational model is based on a bidomain approach to describe the electrical network of cells and a heterogeneously modified Priebe-Beuckelmann human ventricular cardiomyocyte model, which characterizes the electrophysiological properties [4].

The bidomain model represents the current flow through the gap junctions as well as through the intra- and extracellular space [5]. It models the anisotropic electrical behavior of myocardial tissue in two domains. The bidomain model is calculated with help of solving Poisson's equation for stationary electric fields numerically with the finite difference method.

The electrophysiological model of Priebe-Beuckelmann [6] includes a set of nonlinear-coupled first order ordinary differential equations to model intra- and extracellular ion concentrations, ion-flows through the cell membrane, state variables and dynamic changes in ionic channels, description of the behavior of intracellular structures, and the transmembrane voltage (TMV). Varying the channel density of the currents $I_{t o}, I_{K s}, I_{K 1}$, and $I_{N a C a}$ as measured transmurally in ventricular cells establishes electrophysiological heterogeneity [4].

Fig. 1A depicts the variation of the maximum conductances of these channels depending on the position in the left ventricular free wall. The maximum conductances at the epicardial border are set by $g_{t o}=0.5204$ $\mathrm{pA} / \mathrm{pF}, g_{K s}=0.0415 \mathrm{pA} / \mathrm{pF}, g_{K 1}=0.61 \mathrm{pA} / \mathrm{pF}$, and $g_{N a C a}$ $=1000 \mathrm{pA} / \mathrm{pF}$. The physiological action potentials of the three cell types are illustrated e.g. in fig. 3A-C with dashed lines. Fig. 1B shows simulated transmural ECGs of a homogeneous model compared to a heterogeneous one as a verification of the correctness of this approach. Only the heterogeneous model is characterized by a typical positive T wave.

LQT1, LQT2, and LQT3 are incorporated into this
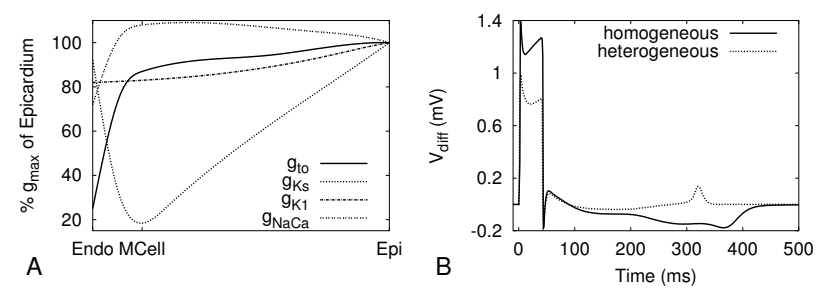

Figure 1. Heterogeneous characteristics of the modified Priebe-Beuckelmann model. A: Distribution of the maximum conductances of $I_{t o}, I_{K s}, I_{K 1}$, and $I_{N a C a}$ regarding the epicardial values. B: Transmural ECGs of a homogeneous and a heterogeneous model. Only the heterogeneous model shows a positive $\mathrm{T}$ wave.

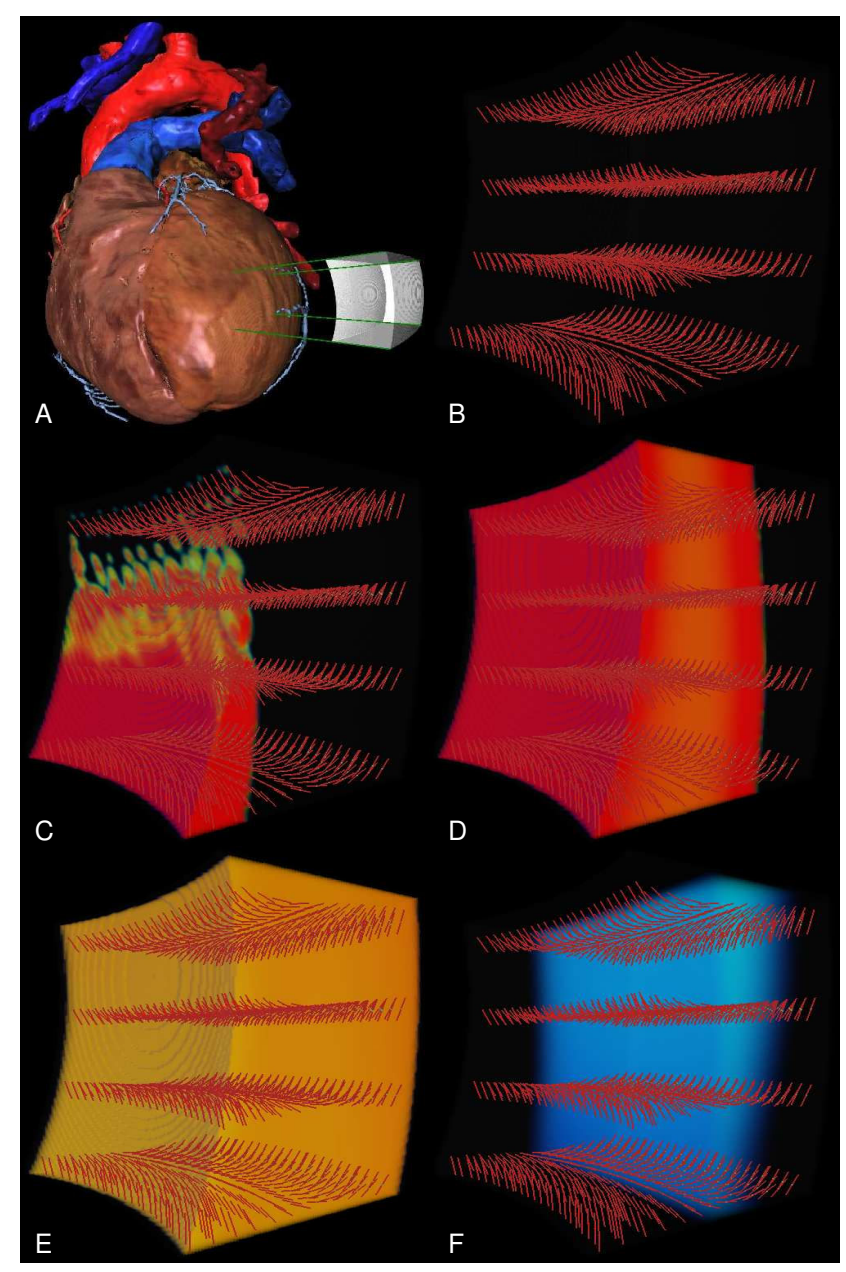

Figure 2. Model of the left ventricular wall. A: Virtual wedge preparation in the anatomical content. B: Fiber orientation inside the model. Fibers are depicted by lines in four exemplary slices. C+D: Color coded transmembrane voltage (TMV) during depolarization. E+F: Color coded TMV during repolarization. The repolarization finally vanishes in the mid-myocardium, which leads to the positive $\mathrm{T}$ wave.

electrophysiological model by changing the ion channel properties of the currents $I_{K s}, I_{K r}$, and $I_{N a, L}$, resp. [7]. The maximum conductance $g_{K s, \max }$ and $g_{K r, \max }$ of $I_{K s}$ and $I_{K r}$ are reduced to characterize LQT1 and LQT2, resp.. A Markov model of $I_{N a}$, which describes a mutation in the $\mathrm{C}$ terminus of the channel called $1795 \mathrm{insD}$ [8], is incorporated to describe LQT3.

The underlying anatomical structure of this model is a virtual left ventricular wedge preparation with surrounding bath medium (see fig. 2A). The model consists of $116 x 134 x 134$ voxels with a side length of $0.2 \mathrm{~mm}$ pre voxel. The orientation of muscle fibers is included using human measurement data [9]. Fig. 2B depicts the 

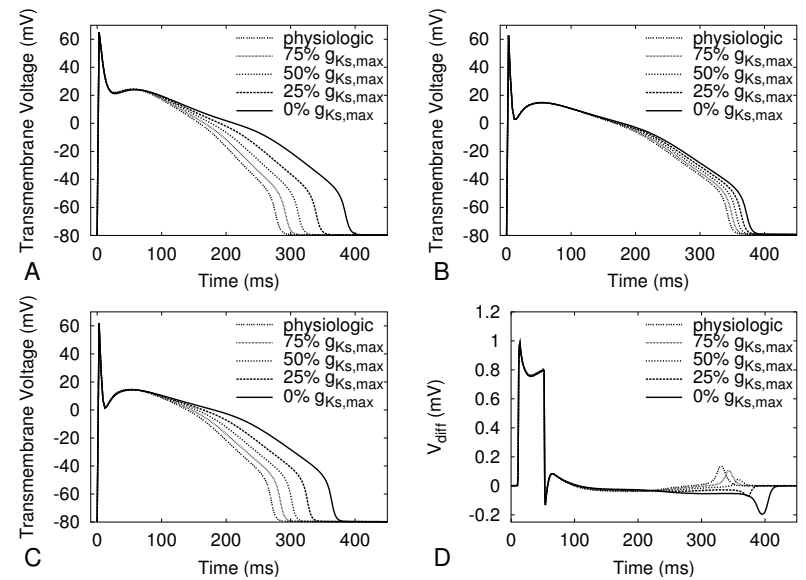

Figure 3. Model of LQT1. Changes of TMV under consideration of the changes of $g_{K s, \max }$ are shown for single (A) endocardial, (B) M, and (C) epicardial cells. D: Transmural ECG.

variation of the helix angle from endocardial $\left(55^{\circ}\right)$ via midmyocardial $\left(0^{\circ}\right)$ to epicardial myocardium $\left(-75^{\circ}\right)$ leading to a anisotropic electrical behavior.

The excitation of the ventricular wall model is initiated at the subendocardial myocardium by applying pointwise intracellular currents. The application of current starts at apical points modeling myocytes with connections to Purkinje fibers and wanders basal.

The two electrodes for calculating the transmural ECG on basis of the gradient of the extracellular potential are placed in the bath medium in the center of the model near to the endo- and epicardium, resp..

\section{Results}

Fig. 2 illustrates the result of a simulation of the physiological excitation propagation in the heterogeneous model. The excitation starts at the endocardial side (fig. 2C) and spreads towards epicardium (fig. 2D). In fig. 2E all cells are in the repolarization phase 2 (plateau phase). The final repolarization starts both in endo- and epicardial areas and vanishes finally in the mid-myocardium (see fig. $2 \mathrm{~F}$ ). The transmural ECG of this simulation is presented in fig. 1B. This coupled simulation and also single cell simulations are used to investigate the effects of the three presented types of LQTS in different stages of the genetic defect.

The effects of the LQT1 syndrome are shown in fig. 3. The reduction of $g_{K s, \max }$ leads to a prolongation of the APD in all three cell types. Other than in measurement data, the APD of the M cells is not increased in the same way as of endo- or epicardial cells. This is provoked by the homogeneously distributed $I_{K r}$ as the remaining repolarization current. This is the reason why the APD of all three cell types is nearly the same for complete loss of $I_{K s}$ function. These properties cause the change in polarization of the $\mathrm{T}$ wave in the transmural ECG (fig. 3D). The differences between experimental and simulated reults can be that a genetic defect of $I_{K s}$ might also influence the properties of $I_{K r}$ or that an additional potassium current is activated due to the genetic defect of $I_{K s}$. Both effects are not considered in this approach. Besides the varying amplitude of the $\mathrm{T}$ wave an increased duration of the QT interval caused by declining density of $I_{K s}$ channels and no widening of the $\mathrm{T}$ wave morphology is visible.

The reduction of $I_{K r}$ channel density as mechanism for the LQT2 syndrome lead in the uncoupled single cell simulations to a prolongation of the APD in all of the three cell types but mostly in $\mathrm{M}$ cells (see fig. 4A-C). This is provoked by the heterogeneously distributed density of $I_{K s}$ as the remaining repolarization current (see fig. 1A). In the transmural ECG (fig. 4D) an extension of the QT interval with an accompanying widening of the $T$ wave and enlarged amplitude, which is caused by the increased APD in midmyocardial areas, is visible during decreasing density of $I_{K r}$ channels.

The LQT3 syndrome is depicted by the inclusion of the presented mutation of $I_{N a}$ channels leading to a late component during the plateau phase. The APD prolongation in the single cell simulations is most dominant in mid-myocardial cells (see fig. 5A-C). This effect happens because the APD of M cells is already longer than in endo- or epicardial cells in the physiological case, which leads to a larger component of the again depolarizing late $I_{N a, L}$ in $\mathrm{M}$ cells. This dispersion of the repolarization is maintaining the increased duration of the QT interval as well as the widening and enlargement of the amplitude
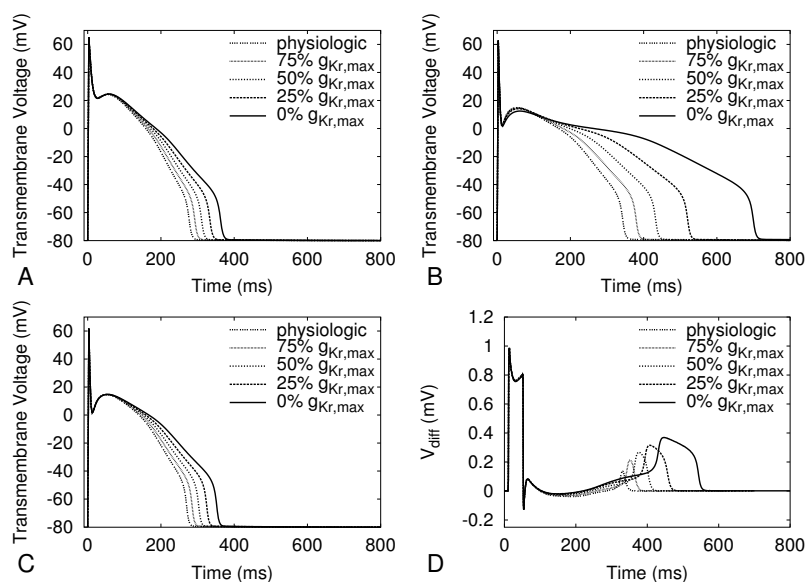

Figure 4. Model of the LQT2 syndrome. A, B, and C show the variation of TMV for single endocardial, $\mathrm{M}$, and epicardial cells during the change of $g_{K r, \max }$, resp.. D: Transmural ECG of five different stages of LQT2. 

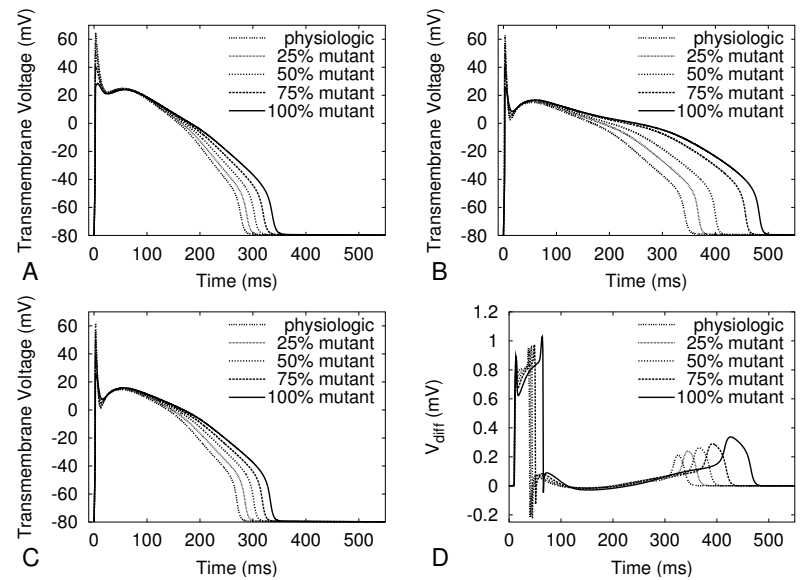

Figure 5. Model of LQT3. TMV variation of (A) endocardial, (B) M, and (C) epicardial cells due to the inclusion factor of mutant $I_{N a}$. D: Transmural ECG. The signal during depolarization phase is getting wider during inclusion of more mutant $I_{N a}$ channels, which is visible in the slowing of the excitation propagation velocity.

of the $\mathrm{T}$ wave (fig. 5D). Besides these effects the speed of the excitation propagation is reduced during increased inclusion of mutant $I_{N a}$ channels. The reason for this is the reduced speed of the depolarization process due to the mutation. The widening of the signal in the ECG during depolarization is visible in fig. 5D.

\section{Conclusion}

The simulation results demonstrate the ionic mechanisms of the three most common LQTS in single cell studies and in a model of a human left ventricular wedge preparation. The effects of the LQTS are presented in transmural ECGs. The morphology of the waveforms is comparable to measured data (see [2] and [3]). Only in the case of LQT1 the model does not represent the correct results but still shows prolongation of the QT interval.

Gima and Rudy recently published similar simulations in a one-dimensional model of a guinea-pig with a simplified electrical heterogeneity [10]. The novelty of our work was to characterize LQTS in a threedimensional anisotropic heterogeneous human model of the left ventricular myocardium using the bidomain approach.

In future, the models have to be enhanced to examine further pathologies. The advances in genetic research will provide the framework to describe genetic and other defects of cardiomyocytes. With such simulations one can predict electro-mechanical properties of diseased hearts that have not been measured yet. To obtain more realistic results for the clinical application, further human electrophysiological measurements has to be included in the models.
The presented electrophysiological model can be combined with a mechanical model to achieve a fully coupled excitation-contraction model of the whole heart, if computer resources and the availability of measurement data will increase as it has in the past decades.

\section{References}

[1] Antzelevitch C, Yan G, Shimizu W, Burashnikov A. Electrical heterogeneity, the ECG, and cardiac arrhythmias. In Zipes DP, Jalife J (eds.), Cardiac Electrophysiology. From Cell to Bedside, 3 edition. Philadelphia: W. B. Saunders Company. ISBN 0-7216-7811-4, 1999; 222-238.

[2] Shimizu W, Antzelevitch C. Cellular basis for the ECG features of the LQT1 form of the long-QT syndrome: Effects of $\beta$-adrenergic agonists and antagonists and sodium channel blockers on transmural dispersion of repolarization and torsade de pointes. Circ 1998;98:2314-2322.

[3] Shimizu W, Antzelevitch C. Sodium channel block with mexiletine is effective in reducing dispersion of repolarization and preventing torsade de pointes in LQT2 and LQT3 models of the long-QT syndrome. Circ 1997; 96:2038-2047.

[4] Seemann G, Sachse FB, Weiß DL, Dössel O. Quantitative reconstruction of cardiac electro-mechanics in human myocardium: Regional heterogeneity. J Cardiovasc Electrophysiol 2003;14(Suppl.). In press.

[5] Henriquez CS, Muzikant AL, Smoak CK. Anisotropy, fi ber curvature and bath loading effects on activation in thin and thick cardiac tissue preparations: Simulations in a threedimensional bidomain model. J Cardiovasc Electrophysiol May 1996;7(5):424-444.

[6] Priebe L, Beuckelmann DJ. Simulation study of cellular electric properties in heart failure. Circ Res 1998;82:12061223.

[7] Viswanathan P, Rudy Y. Cellular arrhythmogenic effects of congenital and acquired long-QT syndrome in the heterogeneous myocardium. Circ 2000;101:1192-1198.

[8] Clancy CE, Rudy Y. $\mathrm{Na}^{+}$channel mutation that causes both brugada and long-QT syndrome phenotypes. Circ 2002; 105:1208-1213.

[9] Streeter DD. Gross morphology and fi ber geometry of the heart. In Bethesda B (ed.), Handbook of Physiology: The Cardiovascular System, volume I. American Physiology Society, 1979; 61-112.

[10] Gima K, Rudy Y. Ionic current basis of electrocardiographic waveforms - A model study. Circ Res 2002;90:889-896.

Address for correspondence:

Gunnar Seemann

Institut für Biomedizinische Technik, Universität Karlsruhe (TH) Kaiserstr. 12 / 76128 Karlsruhe / Germany

tel./fax: ++49-721-608-2790/2789

Gunnar.Seemann@ibt.uni-karlsruhe.de 